\title{
Generalized phase stability in multipacting
}

\author{
Valery Shemelin* \\ Cornell Laboratory for Accelerator-based Sciences and Education (CLASSE), Ithaca, New York 14853, USA
}

(Received 24 June 2011; published 20 September 2011)

\begin{abstract}
Analysis of known experimental data for the boundaries of the multipactor discharge in a flat gap shows that these data cannot be explained in the frames of a simple two-point theory. More complicated theories include repeating impacts of the electrons on the same surface before they hit the opposite one. Here an approach is proposed that can illustrate many of these theories: a phase map connecting the start and arrival phases of the electrons coming to the same or opposite plate. This illustrative technique leads to a concept of a generalized phase stability. Introduction of the generalized condition of stability helps to understand the expanding of multipacting zones beyond the limits predicted by the simple stability condition. The phase diagrams illustrate the phase motion when the simple condition of stability is applicable or not applicable.
\end{abstract}

DOI: 10.1103/PhysRevSTAB.14.092002

PACS numbers: 52.80.Pi, 79.20.Hx

\section{INTRODUCTION}

The first two-point theory of the multipactor discharge in a flat gap [1,2] explains existence of borders of multipacting bands, or zones. This theory uses an assumption introduced by Gill and von Engel [3] that a parameter $k$ equal to the impact velocity of the primary electrons relative to the emission velocities of secondaries is constant.

This nonphysical assumption was replaced with the more realistic assumption of a nonzero initial velocity not directly related to the velocity of the primary electrons $[4,5]$. This velocity can fluctuate and a condition of stability was derived for these fluctuations [4]. The stability condition (or phase focusing) is introduced in [5] also, not specifically for velocity deviations but for any phase deviations. Moreover, this condition is used for an estimation of the maximal charge density in a flat sheet of multipacting electrons.

However, even after these publications, new papers appeared [6,7] further developing the concept of the constant $k$.

Asymmetric back-and-force orbits expand the zones of the multipactor; they were studied in several works $[6,8]$.

Further, more complicated physical situations were analyzed for multipacting: coaxial electrodes along with the flat gap geometry [9], usage of biases with DC electric and magnetic fields [9-11], the flat gap case was extended to the rectangular waveguide geometry [12], and the rectangular waveguide was compared with the wedgelike waveguide [13-15].

Another interesting case of the multipactor in a flat gap is when one of the plates is a dielectric [16]. In this case the

\footnotetext{
*vs65@cornell.edu
}

Published by the American Physical Society under the terms of the Creative Commons Attribution 3.0 License. Further distribution of this work must maintain attribution to the author(s) and the published article's title, journal citation, and DOI.
DC bias can be caused both by negatively and positively charging dielectric and the discharge can cease when the dielectric is charged.

Some authors pay special attention to the properties of the secondary electron emission taking into account not only the value of the secondary emission yield (SEY) but also energy distribution of primary electrons [9], the random character of the secondary electron velocities, and delay time of the secondary emission [8]. Influence of the energy and impact angle dependencies of the SEY on the multipactor boundaries, also as input of true secondary, elastically, and not elastically reflected electrons, is analyzed in [17-19]. With a high enough SEY hybrid resonant mode can transit to the polyphase regime [20-22].

The models including the realistic properties of the electron emission should include statistical methods [23] and require numerical simulations.

We are not going to refer here to the numerical codes which are described in many papers. Multipactor in more complex geometries (coaxial lines, waveguides with partly reflected power, resonant cavities, etc.) are beyond the scope of this short overview. We do not discuss the saturation level of the multipactor discharge. Let us mention only one more focusing mechanism in a flat gap whereby the leading edge of the discharge grows at the expense of the trailing edge that may shape very tight sheets of electrons in a steady state [24]. More detailed though also a concise historical review of the multipactor discharge is presented in [25].

The papers cited above relate mainly to a multipactor in a flat gap because in the following we want to compare the analytical results with the experimental data for a flat gap and propose a pictorial model illustrating some specific properties of the phenomenon.

In the following, the analytical description of the multipacting $[4,26]$ is used as a starting point. The influence of the normal and tangential components of the initial velocity 


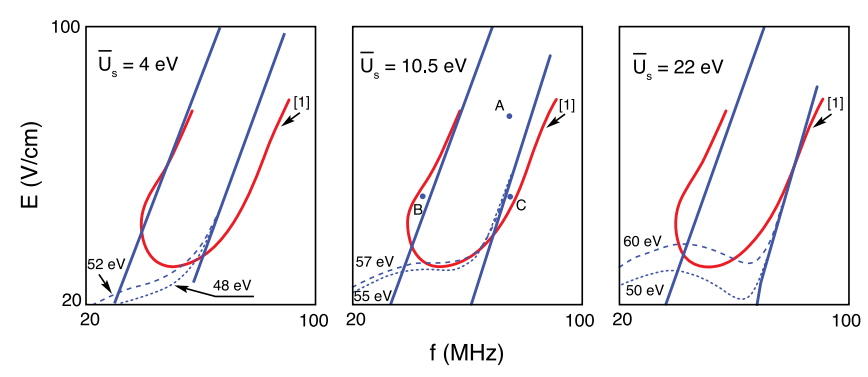

FIG. 1. Experimental curve [1] and calculated borders of the multipactor zone for different values of the initial energy $\bar{U}_{s}$ of secondary electrons. Electrode separation is $3 \mathrm{~cm}$. The straight solid lines are obtained from the condition of stability, dashed and dotted lines correspond to a border with $\mathrm{SEY}=1$ with different impact energy.

on the position of discharge bands and some considerations for those starting phases when the decelerating field can return the electron to the same electrode from where it started are taken into account. Yet the difference between calculated and measured borders has remained significant. Of course, more complicated kinematic solutions from the papers cited above can be used. However, we will show a somehow different presentation.

As an example of such a discrepancy between simple analytical theory and reality, we will analyze the experimental results of a pioneer paper by Hatch and Williams [1] taking into account conditions of stability and limitations by low impact energy. In the original work the condition of stability was presented for the motion that looked like a reflection of electrons from the walls with a certain coefficient of elasticity. But such a reflection does not correspond to the experimental data. The value of the coefficient was just fitted to the experiment.

On the experimental zone of multipacting, we will consider three points: $A, B$, and $C$ (Fig. 1) where the discharge was observed. The earlier theory $[4,26]$, without improvement for more complicated trajectories as pointed above, explains existence of discharge only in $A$.

In the present article generalization of the phase stability condition is proposed. The revised stability condition gives a better understanding of the experimental results.

\section{ANALYSIS OF THE EQUATION OF MOTION}

The equation of motion for an electron in the gap is

$$
\ddot{y}=\frac{e U}{m d} \sin \omega t,
$$

where the coordinate $y$ is measured normal to the surface of one of the electrodes; $\mathrm{e} / \mathrm{m}$ is the specific charge of the electron, the charge is considered positive to simplify the writing; $U$ is the voltage across the gap, which is of length $d ; \omega=2 \pi f$, where $f$ is the oscillation frequency; $t$ is the time. It is helpful to rewrite this in the normalized form:

$$
\lambda^{\prime \prime}=\xi \sin \theta
$$

where $\lambda=x / d, \xi=U / U_{0}, U_{0}=m \omega^{2} d^{2} / e$, and $\theta=\omega t$; primes denote derivatives with respect to $\theta$, while dots indicate derivatives with respect to $t$.

Integrating Eq. (1), we obtain

$$
\begin{gathered}
\lambda^{\prime}=\xi\left(\cos \theta_{1}-\cos \theta\right)+\beta_{1}, \\
\lambda=\xi\left(\theta-\theta_{1}\right) \cos \theta_{1}+\xi\left(\sin \theta_{1}-\sin \theta\right)+\beta_{1}\left(\theta-\theta_{1}\right) .
\end{gathered}
$$

Here $\theta_{1}$ is the phase at which the electron enters the gap, and $\beta_{1}=v_{\perp} / \omega d$ is the dimensionless normal component of the initial velocity of the secondary electron.

As it is shown in [4,26], the value of $\beta_{1}$ corresponds approximately to one-half of the initial energy of secondary electrons and can be expressed as follows:

$$
\beta=\frac{\bar{v}_{\perp}}{\omega d}=\frac{2}{3} \frac{\bar{v}}{\omega d}=\frac{2}{3} \sqrt{\frac{2 \bar{U}_{s}}{U_{0}}} .
$$

Here the factor of $2 / 3$ appears because of the angular distribution of the secondary electrons and $(2 / 3)^{2} \approx 0.5$ leads from $\bar{U}_{s}$ to $\sim 0.5 \bar{U}_{s}$ for the normal component.

The condition for the electron to "resonantly" cross the gap is that the transit time be equal to an odd multiple of half periods of the rf field; this ensures that newly generated secondary electrons see the same relative phase of the field as their predecessors. Equation (3) implies that

$1=\xi\left(\theta_{2}-\theta_{1}\right) \cos \theta_{1}+\xi\left(\sin \theta_{1}-\sin \theta_{2}\right)+\beta_{1}\left(\theta_{2}-\theta_{1}\right)$,

where $\theta_{2}$ is the phase at which the electron reaches the second electrode at $\lambda=1$. Since the transit time $\theta_{2}-\theta_{1}=(2 n-1) \pi,(5)$ gives

$$
\xi=\frac{1-(2 n-1) \pi \beta_{1}}{(2 n-1) \pi \cos \theta_{1}+2 \sin \theta_{1}} .
$$

So, for each starting phase $\theta_{1}$ we can find the value of normalized voltage $\xi$ and hence voltage $U$ across the gap. The discharge is possible at this voltage if the stability condition is satisfied. Usually, this means that the initial phase of next generations of electrons does not change significantly if the initial electron has an excursion of its phase. The excursion of the phase relative to the equilibrium phase will decrease with each crossing the gap under the condition

$$
\left|\partial \theta_{2} / \partial \theta_{1}\right|<1 .
$$

We will call this the simple stability condition. Later we will introduce the conception of a generalized stability condition.

Analysis of the motion for the marked points $A, B$, and $C$ could be performed right away; however, we need to pay attention to the value $\beta_{1}$ defined earlier.

The most probable initial velocity of the secondary electron is usually associated with the peak of energy distribution of these electrons. However, it is wrong, and 
because the initial energy $\bar{U}_{s}$ defines the value $\beta_{1}$, we will discuss this separately.

\section{DISTRIBUTION OF INITIAL VELOCITIES AND THE BOUNDARY WITH SEY $=1$}

In the discussed experiment silver-plated electrodes were used. The energy distribution of secondary electrons for the case of silver electrodes can be taken from [27]. The expression for the energy distribution function $d n=f(W) d W$ can be recalculated for initial velocities or, which is more convenient, for the variable of $\sqrt{W}$ :

$$
d n=f\left(m v^{2} / 2\right) m v d v=2 \sqrt{W} f(W) d \sqrt{W}
$$

As seen from Fig. 2, the distribution with respect to $\sqrt{W}$ has a well-defined maximum corresponding to the most probable velocity of the secondary electrons.

For calculation of this distribution, the original experimental data (Fig. 2, upper left) were presented analytically as

$$
\begin{aligned}
f(W)= & 0.07+p \cdot \exp \left(-\frac{W^{n}}{A}\right) \\
& -(p+0.07) \cdot \exp \left(\frac{-W-0.05 W^{2}}{B}\right)
\end{aligned}
$$

with $p=1.15, n=1.05, A=22$, and $B=3$. This gives the average value of the secondary electron energy as $22 \mathrm{eV}$ and the most probable velocity corresponds to energy of $10.5 \mathrm{eV}$. We neglect here the elastically scattered electrons, which give the sharp maximum in Fig. 2, upper left picture.
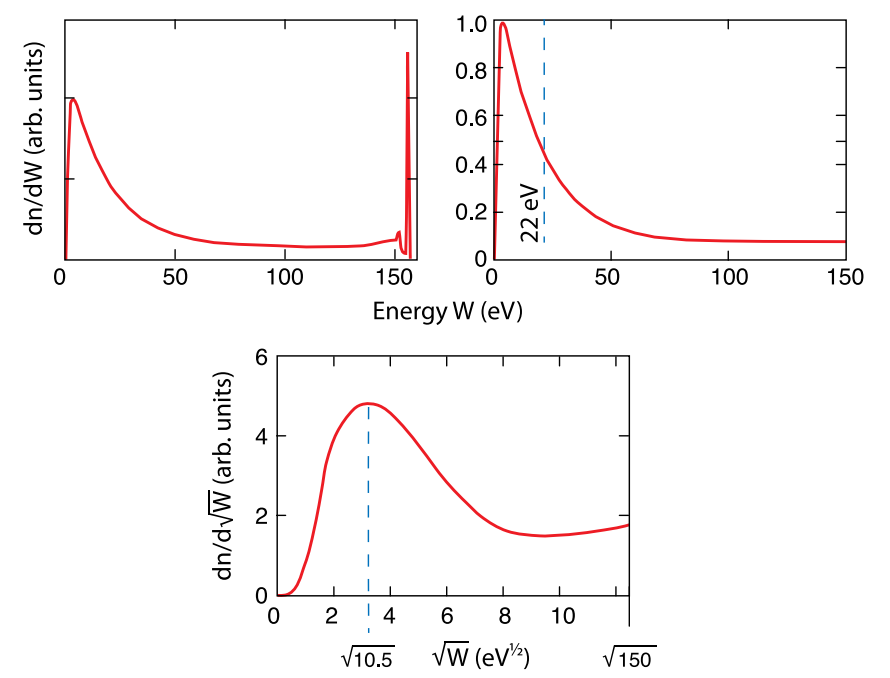

FIG. 2. Energy distribution of secondary electrons emitted by silver [27], its analytically obtained approximation, and distribution with respect to square root of energy. Energy of primary electrons is about $150 \mathrm{eV}$.
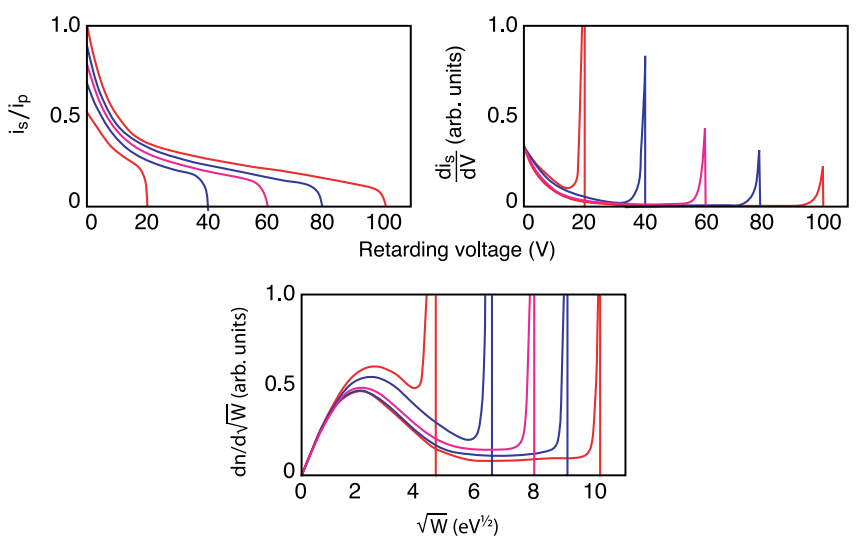

FIG. 3. Energy distribution of secondary electrons emitted by copper [27], its analytically obtained derivative, and distribution with respect to square root of energy. Energy of primary electrons is from 20 to $100 \mathrm{eV}$.

In Fig. 1 the upper and lower borders of the 1 st $(n=1)$ discharge zone are presented for different values of the initial energy (straight lines). These borders are obtained in [4] from condition (7). Also shown are the curves for the energy bounds corresponding to $\mathrm{SEY}=1$. One can see that the curvature of the line showing the energy bound fits experimental data best for the initial energy of $10.5 \mathrm{eV}$, i.e., for the most probable velocity. The initial energy of secondary electrons of $4 \mathrm{eV}$ used in earlier papers $[4,26]$ is presumably not consistent with this physical situation (see Fig. 1, left) though gives maximum in the energy distribution. The average value of energy, $22 \mathrm{eV}$ (Fig. 1, right), also cannot be used.

However, the initial energy value $\bar{U}_{s}=4 \mathrm{eV}$ appears to be correct if we analyze the copper electrodes. In Fig. 3, upper left, the experimental data [27] for copper are shown. The next two pictures present the mathematical handling of these data. It is clearly seen that, for the initial energy of primary electrons $20-100 \mathrm{eV}$, the velocity peak position is close to $4-6 \mathrm{eV}$. This data for copper (Fig. 3, upper right) reveals a peak of energy distribution at $V=0$, not at $V=4-6$ volts as it is for silver (Fig. 2, both upper pictures), and the peak of reflected electrons is too broad in comparison with the previous data for silver. Both these features are determined by derivatives at the ends of curves. However, even if there are some experimental errors, the peak of velocity distribution at $4 \mathrm{eV}$ is determined by the behavior of the middle part of these curves and can be treated as more or less reliable.

\section{STABILITY CONDITION FOR DIFFERENT POINTS OF THE MULTIPACTING ZONE}

The condition for the stable electron motion in a multipactor discharge requires that the electron enter the gap at a definite phase. We can use (6) to calculate the normalized voltage at which the discharge exists for a given start phase. 


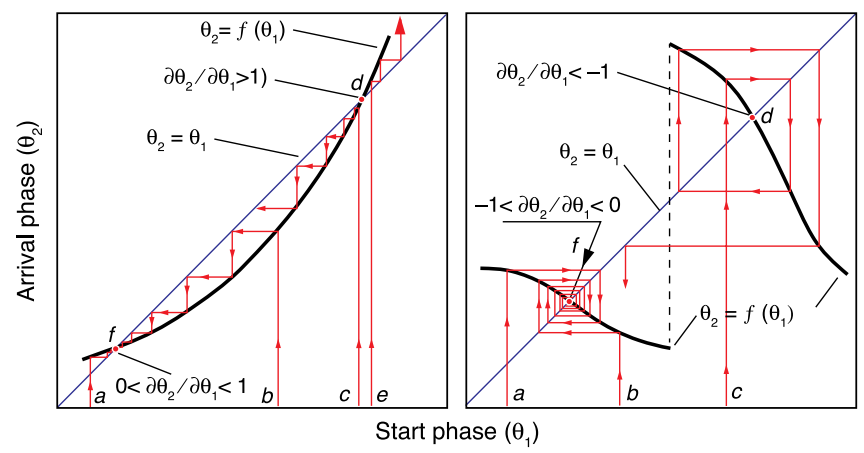

FIG. 4. Focusing to a stable phase and defocusing from an unstable phase for an increasing and a decreasing function.

The change in the phase at the exit from the gap is determined by fluctuations in the initial velocity and by the initial change in the phase at the entrance. Here we will neglect the initial velocity fluctuation: it is shown [3] that the same results are obtained if we take into account fluctuations in the initial phase only.

If the particle crosses the gap, the function $\theta_{2}=f\left(\theta_{1}\right)$ can be obtained from (5). If the electron goes back to the same surface where it was emitted, the value of 1 in the left-hand side of (5) should be replaced by 0 .

Let us introduce a simple graphic interpretation of phase motion, focusing and defocusing. With the help of a bisector of the right angle between coordinate axes, we can easily find the starting phase for the next gap crossing if we know the previous starting phase and if the function $\theta_{2}=f\left(\theta_{1}\right)$ is defined.

The examples of phase motion of an electron in the gap are shown in Fig. 4. Four different cases of the value $\partial \theta_{2} / \partial \theta_{1}$ used in (7) are shown in the picture. It is arbitrarily assumed for this figure that the secondary electron yield $\mathrm{SEY}=1$. Focusing $(f)$ and defocusing $(d)$ points are shown for positive (left picture) and negative (right) values of $\partial \theta_{2} / \partial \theta_{1}$.

Let us subtract the integer odd number of $\pi$ radians from the phase $\theta_{2}$ if the particle crosses the gap and subtract the even number of $\pi$ radians from this phase if the particle falls on the same electrode it was emitted from. Let these integer numbers be such that the phases of arrival are in the interval $[-\pi, \pi]$. Then the phase of the particle "in resonance" will be the same after crossing the gap: $\theta_{2}=\theta_{1}$. It is obvious that the condition of stability (7) will not change after such a transformation of $\theta_{2}$. The advantage of this transformation lies in the fact that both phases, $\theta_{1}$ and $\theta_{2}$, are now placed on the limited intervals, and the graphical interpretation of the phase motion becomes very illustrative.

Now, when we have defined the value of $\beta_{1}$ and redefined $\theta_{2}$, we can return to construction of the function $\theta_{2}=$ $f\left(\theta_{1}\right)$ for the points $A, B$, and $C$ of Fig. 1. This function, the phase trajectory for an arbitrary initial starting phase, and the corresponding impact energy are presented in Fig. 5. For each of the three points 100 flights of the particle are
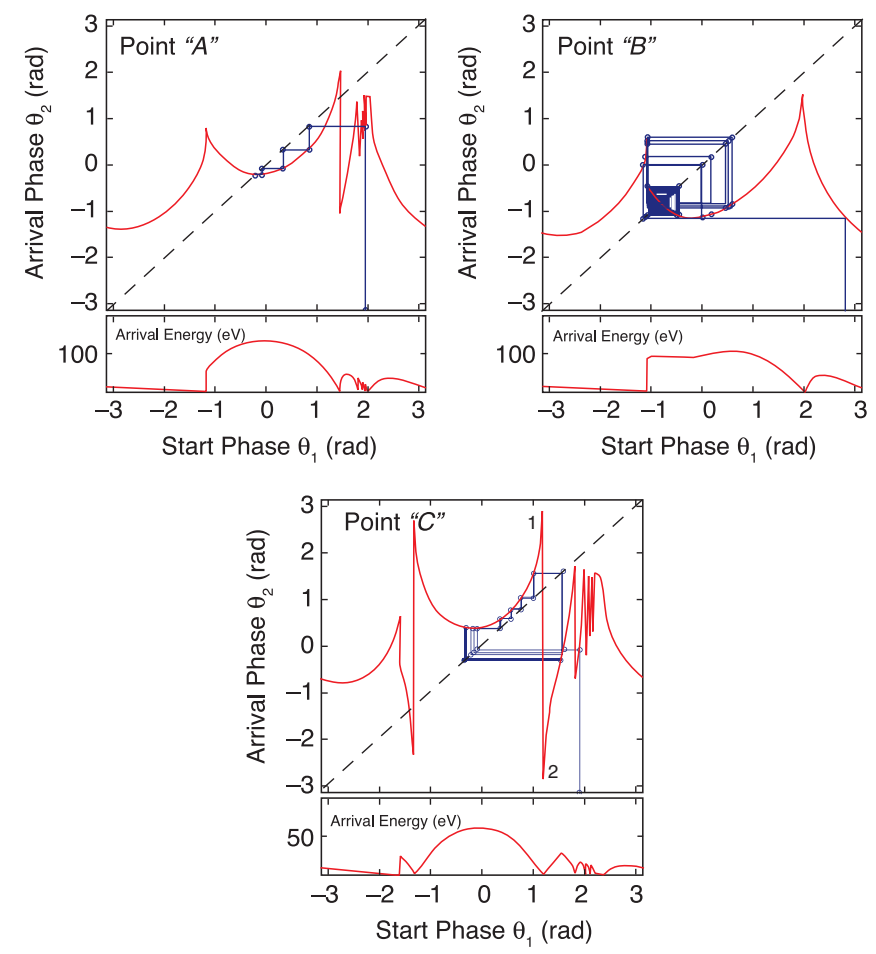

FIG. 5. Starting and arrival phases, and impact energy at arrival for the marked points of Fig. 1.

calculated. It is assumed again that neither generation of new electrons nor loss of them occurs on the surface $(\mathrm{SEY}=1)$.

The case of point $A$ is very simple. The condition (7) is satisfied for $\theta_{1}=-0.227$. After five crossings of the gap the particle settles down to the focus point and all next phase positions coincide. The impact energy at this point is high enough, about $130 \mathrm{eV}$ (see the lower part of the picture), to produce secondary electrons with SEY $>1$. So it is a point of multipacting.

The simple stability condition is not satisfied in the point $B$ because $\partial \theta_{2} / \partial \theta_{1}=-1.09<-1$. Let us distinguish two squares on the picture for this case. The inner one is tightly hatched because though the defocusing process develops, the value of the derivative is not too high and lines go close one to another. The other one is the maximal square on the bisector the upper side of which is shown in Fig. 5 for the point $B[M N P Q$ in Fig. 6(a)]. The pattern of (a)

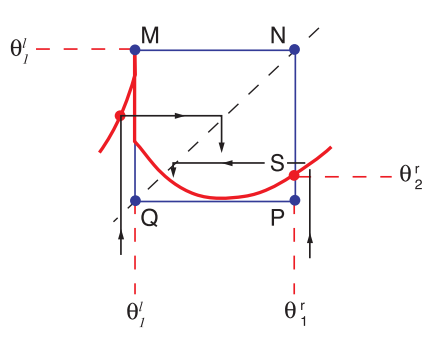

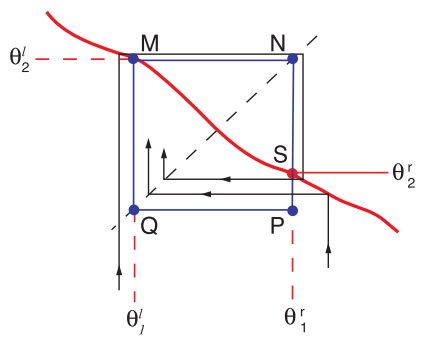

(b)
FIG. 6. A square of stability for the case $B$. 

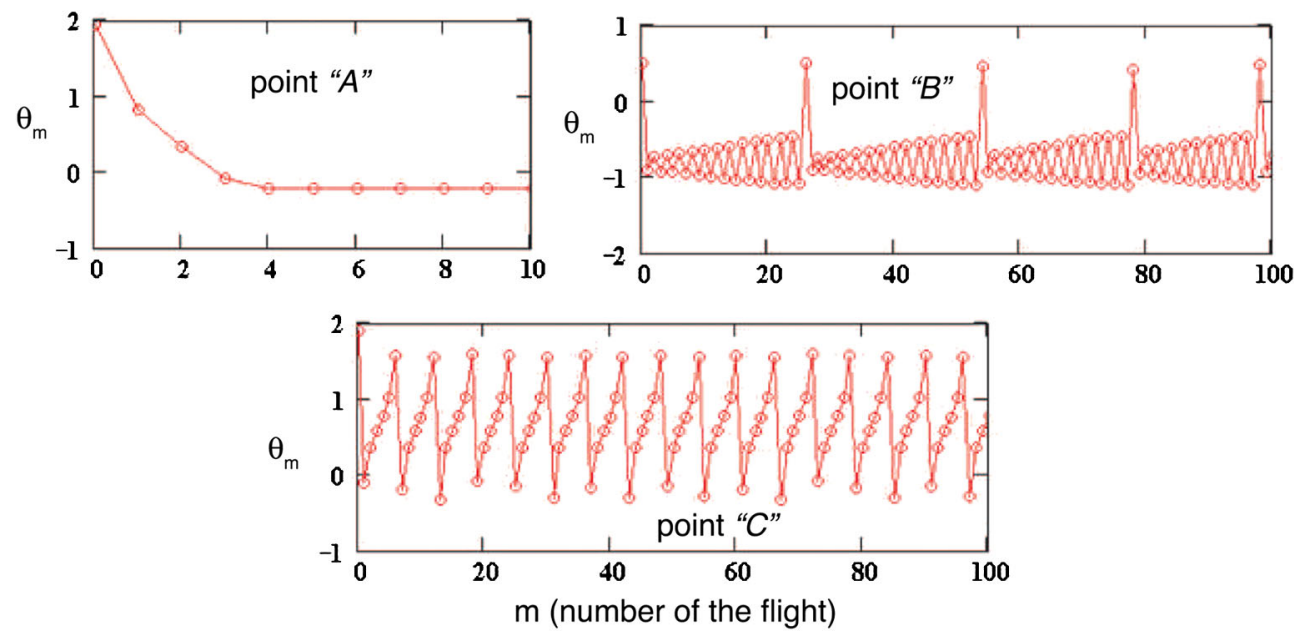

FIG. 7. Dependence of the starting phase on the number of the flight.

the phase trajectory practically does not depend on the initial start phase. When the phase trajectory reaches the point $M$ [see details in Fig. 6(a)], its continuation enters again into the smaller square. This trajectory cannot quit the square $M N P Q$ as can be seen from Fig. 6(a), because after any small deviation of the trajectory outside the square, it enters again into this square. The necessary condition for this is that the entire curve is inside the square. In Fig. 6(b) another possible behavior of the function $f\left(\theta_{1}\right)$ is shown: without a jump of the derivative at $\theta_{1}^{l}$. We can formulate the 1 st condition of the generalized stability: if a part of the curve $\theta_{2}=f\left(\theta_{1}\right)$ can be placed inside a square located on the bisector of coordinate axes as on the diagonal, and the values corresponding to the left and right sides of this square are $\theta_{1}^{l}$ and $\theta_{1}^{r}$, and the related values $\theta_{2}^{l}$ and $\theta_{2}^{r}$ satisfy the ratio

$$
\left|\frac{\theta_{2}^{r}-\theta_{2}^{l}}{\theta_{2}^{r}-\theta_{2}^{l}}\right|<1
$$

the motion is finite and limited by phase angles $\theta_{1}^{l}$ and $\theta_{1}^{r}$. The simple condition of stability (7), as can be easily seen, is a particular case of (8).

The whole curve defined on the interval always satisfies condition (8) but it does not make a special physical sense, just presents another limiting case.

The case of point $C$ is a case of a repetitive motion when part of the curve escapes from the square (peaks 1 and 2, Fig. 5) but the whole trajectory stays within a limited interval. For description of this motion let us introduce "higher order" functions:

$$
\begin{aligned}
\theta_{3} & =f_{2}\left(\theta_{1}\right)=f\left[f\left(\theta_{1}\right)\right], \\
\theta_{4} & =f_{3}\left(\theta_{1}\right)=f\left[f_{2}(\theta 1)\right], \ldots, \\
\theta_{m+1} & =f_{m}\left(\theta_{1}\right)=f\left[f_{m-1}\left(\theta_{1}\right)\right] .
\end{aligned}
$$

It appears for the motion presented in Fig. 5, point $C$, that the equation

$$
f_{m}\left(\theta_{1}\right)=\theta_{1}
$$

has a solution for $m=6$. So the motion repeats after six different start phases. This motion could be stable if

$$
\left|\partial f_{m}\left(\theta_{1}\right) / \partial \theta_{1}\right|<1
$$

$m=6$ for the point $C$. However, the inequality (11) is not satisfied in this case: $\partial \theta_{7} / \partial\left(\theta_{1}\right)=-3.86$. Nevertheless, because of the particular pattern of the phase trajectory, no particle falls into the region of peaks 1 and 2 (see Fig. 5, point $C$ ). For the outlined square the same condition (8) is valid as for point $B$.

The inequality (11) together with condition (10) can be treated as the second generalized condition of stability. It reduces to the case of the simple stability condition (7), when $m$ is equal to 1 . We should assume $f_{1}\left(\theta_{1}\right) \equiv f\left(\theta_{1}\right)$ in this case.
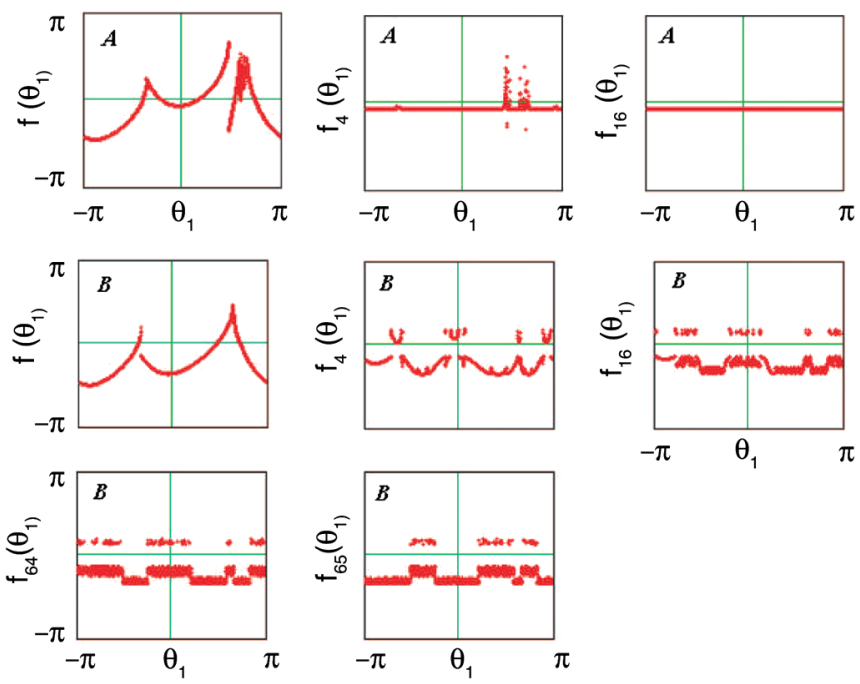

FIG. 8. Phase portrait of multipacting for points $A$ and $B$ of Fig. 1 after different number of flights. 

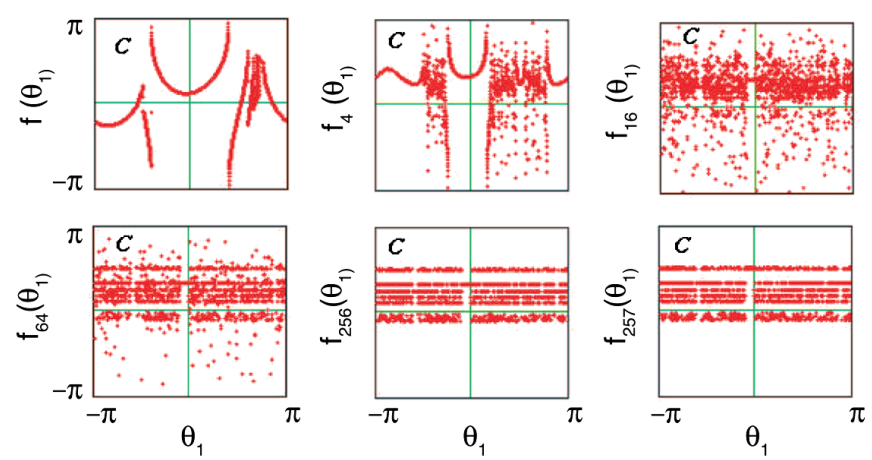

FIG. 9. Same as Fig. 8 but for the point $C$.

Lower parts of the pictures in Fig. 5 show the energy of primary electrons vs the start phase. If this energy is too low the discharge can survive only if the product of the SEY for all the cases of impact keeps bigger than unity. As can be seen from the data in [19] even at very low energies the value of the SEY does not drop below approximately 0.7 due to elastically scattered electrons and the discharge can persist.

Another presentation of the phase motion is shown in Fig. 7. Here the starting phase $\theta_{m}$ is plotted versus the number of the flight.

One more illustration of the grouping process is shown in Figs. 8 and 9. Here for the initial phase $\theta_{1}$ homogeneously distributed in the interval (1000 points), the next generations of particles are shown after different number of flights. Two last pictures for the points $B$ and $C$ show a change of the phase for two successive flights.

\section{CONCLUSIONS}

Introduction of the generalized condition of stability in multipacting helps to understand the expanding of multipacting zones beyond the limits predicted by the simple stability condition. The illustrative phase diagram for a flat gap shows the phase motion when the simple condition of stability is both applicable and not applicable for description of motion. Because of assumption of a yield of 1, stable trajectories may not necessarily lead to growth, a condition of a cumulative SEY $>1$ should be met after each flight in this model.

Other presentations of the grouping process show that the electrons of the multipactor discharge can group into layers even when the simple condition of stability does not work.

The results of this work are applicable for more complicated geometries when experimental zones appear broader than obtained by simulations.
Results obtained for initial velocities of secondary electrons show that the most probable initial velocity of the secondary electrons does not correspond to the most probable their energy, the fact that should be taken into account in simulations.

The author is grateful to Sergey Belomestnykh for useful discussions.

[1] A. J. Hatch and H. B. Williams, J. Appl. Phys. 25, 417 (1954).

[2] A. J. Hatch and H. B. Williams, Phys. Rev. 112, 681 (1958).

[3] E. W. B. Gill and A. von Engel, Proc. R. Soc. A 192, 446 (1948).

[4] V. D. Shemelin, Zh. Tekh. Fiz. 56, 1730 (1986); Sov. Phys. Tech. Phys. 31, 9 (1986).

[5] J. R. M. Vaughan, IEEE Trans. Electron Devices 35, 1172 (1988).

[6] A. L. Gilardini, J. Appl. Phys. 71, 4629 (1992).

[7] A. L. Gilardini, J. Appl. Phys. 78, 783 (1995).

[8] S. Riyopulos, Phys. Plasmas 14, 112101 (2007).

[9] K. Sakamoto et al., J. Phys. D 22, 1840 (1989).

[10] S. Riyopulos et al., IEEE Trans. Electron Devices 44, 489 (1997).

[11] V. Semenov et al., Phys. Plasmas 12, 073508 (2005).

[12] V. Semenov et al., Phys. Plasmas 14, 033501 (2007).

[13] E. Chojnacki, Phys. Rev. ST Accel. Beams 3, 032001 (2000).

[14] V. Semenov et al., IEEE Trans. Plasma Sci. 36, 488 (2008).

[15] J. Hueso et al., IEEE Trans. Electron Devices 57, 3508 (2010).

[16] G. Torregrosa et al., IEEE Electron Device Lett. 27, 619 (2006).

[17] V. P. Gopinath et al., Phys. Plasmas 5, 1535 (1998).

[18] A. Dexter and R. Seviour, J. Phys. D 38, 1383 (2005).

[19] J. de Lara et al., IEEE Trans. Plasma Sci. 34, 476 (2006).

[20] G. S. Luk'yanchikov, Sov. Phys. Tech. Phys. 19, 1196 (1975)

[21] A. Kryazhev et al., Phys. Plasmas 9, 4736 (2002).

[22] I. A. Kossyi et al., J. Phys. D 41, 065203 (2008).

[23] A. Sazontov et al., Phys. Plasmas 12, 053102 (2005).

[24] R. A. Kishek and Y. Y. Lau, Phys. Plasmas 3, 1481 (1996).

[25] R. A. Kishek et al., Phys. Plasmas 5, 2120 (1998).

[26] V. D. Shemelin, Cornell University LNS Report No. SRF 010322-03, 2001.

[27] H. Bruining, Physics and Applications of Secondary Electron Emission (Pergamon Press, London, 1954). 